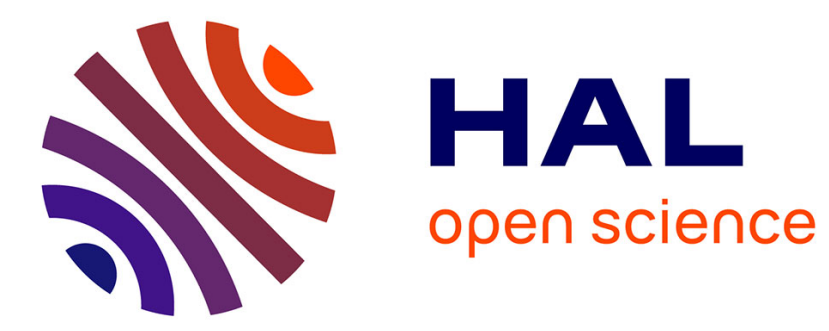

\title{
Optical Control of the Topology of Laser-Plasma Accelerators
}

\author{
J. Vieira, J. T. Mendonça, F. Quéré
}

\section{To cite this version:}

J. Vieira, J. T. Mendonça, F. Quéré. Optical Control of the Topology of Laser-Plasma Accelerators. Physical Review Letters, 2018, 121 (5), pp.054801. 10.1103/PhysRevLett.121.054801 . cea-02277452

\section{HAL Id: cea-02277452 https://hal-cea.archives-ouvertes.fr/cea-02277452}

Submitted on 3 Sep 2019

HAL is a multi-disciplinary open access archive for the deposit and dissemination of scientific research documents, whether they are published or not. The documents may come from teaching and research institutions in France or abroad, or from public or private research centers.
L'archive ouverte pluridisciplinaire HAL, est destinée au dépôt et à la diffusion de documents scientifiques de niveau recherche, publiés ou non, émanant des établissements d'enseignement et de recherche français ou étrangers, des laboratoires publics ou privés. 


\title{
Optical Control of the Topology of Laser-Plasma Accelerators
}

\author{
J. Vieira, ${ }^{1}$ J. T. Mendonça, ${ }^{1}$ and F. Quéré ${ }^{2}$ \\ ${ }^{1}$ GoLP/Instituto de Plasmas e Fusão Nuclear, Instituto Superior Técnico, Universidade de Lisboa, 1049-001 Lisbon, Portugal \\ ${ }^{2}$ LIDYL, CEA, CNRS, Université Paris-Saclay, CEA Saclay, 91191 Gif-sur-Yvette, France
}

(Received 28 February 2018; published 30 July 2018)

\begin{abstract}
We propose a twisted plasma accelerator capable of generating relativistic electron vortex beams with helical current profiles. The angular momentum of these vortex bunches is quantized, dominates their transverse motion, and results in spiraling particle trajectories around the twisted wakefield. We focus on a laser wakefield acceleration scenario, driven by a laser beam with a helical spatiotemporal intensity profile, also known as a light spring. We find that these light springs can rotate as they excite the twisted plasma wakefield, providing a new mechanism to control the twisted wakefield phase velocity and enhance energy gain and trapping efficiency beyond planar wakefields.
\end{abstract}

DOI: 10.1103/PhysRevLett.121.054801

Using plasmas to accelerate particles to high energies has long been identified as a promising path to obtain compact accelerators [1-3]. In terms of particle energy, the most advanced scheme to date for electrons consists in using ultraintense femtosecond lasers or electron beam drivers to excite high-amplitude ultrarelativistic waves in low-density plasmas [4-7]. Electrons trapped into these waves can gain energy and be accelerated to relativistic velocities [8-12].

Currently, longitudinal phase-space properties of the accelerated bunches, such as their longitudinal momentum, can be effectively controlled. Despite recent progress to control the radial dynamics of accelerated beams, using sophisticated temporal wakefield modulations [13] and spatial shapes [14,15], it is not yet possible to control the angular momentum degrees of freedom (d.o.f.) in plasma accelerators. Accessing them is, however, interesting from a fundamental perspective and important for applications, such as radiation generation, which often rely on transverse beam phase space features.

Here, using theory and particle-in-cell (PIC) simulations, we propose to control the angular momentum d.o.f. of relativistic beams by introducing the concept of helicalbeam plasma wakefield accelerators. The helical-beam wakefield accelerator relies on plasma wakefields with orbital angular momentum (OAM). We show that twisted wakefields can generate and accelerate relativistic vortex beams, which, unexpectedly, carry quantized levels of angular momentum. The twisted wakefields could be excited by laser pulses or particle beams with helical profiles. Here, we focus on the laser wakefield accelerator scenario, excited by spatiotemporally shaped femtosecond laser beams called light springs [16] (LS). Light springs have a helical intensity profile, carry OAM, and can effectively transfer angular momentum to the plasma wave. We demonstrate that the LS rotates as it propagates through the plasma, providing an all-optical mechanism to control the wakefield phase velocity, prolong dephasing, and enhance the energy gain in comparison to a planar wakefield.

A pure Laguerre-Gaussian (LG) laser beam with OAM [17] cannot transfer its OAM to a plasma wakefield because the mechanism underlying wakefield excitation is stimulated Raman scattering [18]: since the absorbed and emitted photons each carry the same OAM $\ell_{0} \hbar$, there is no net transfer of OAM to the excited medium [Figs. 1(a) and 1(b)]. Such a transfer becomes possible when the OAM per photon in the laser driver is frequency dependent [Fig. 1(c)]: this corresponds to a spatiospectrally coupled laser beam, where each frequency is associated to a spatial LG mode with a different azimuthal index, $\ell=\ell(\omega)$.
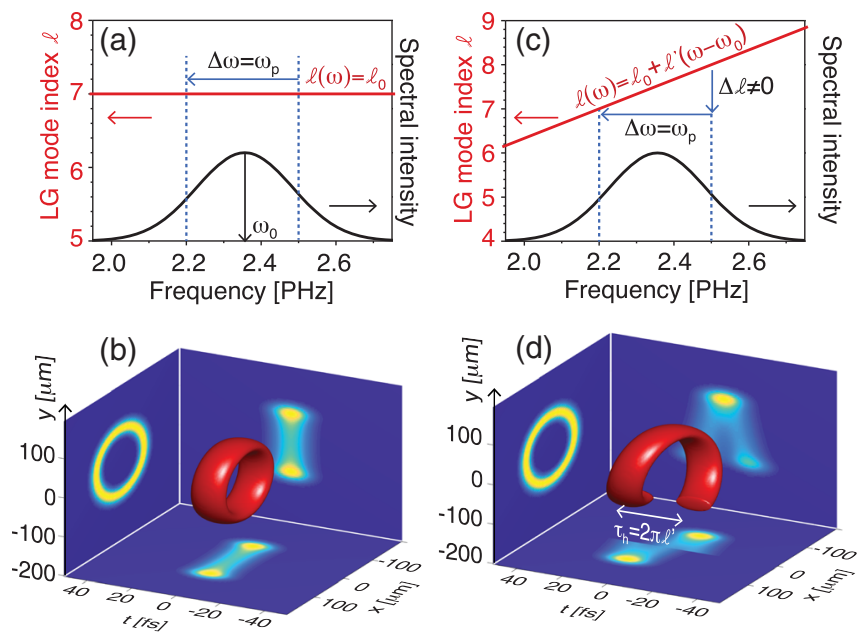

FIG. 1. Pulsed LG beams (a)-(b) versus light springs (c)-(d). The upper graphs show the frequency dependence of the azimuthal mode index $\ell$ in the two cases. The lower graphs display the corresponding spatiotemporal intensity profiles of the pulses. 
When $\ell(\omega)$ is linear, such a superposition of modes forms a LS [Fig. 1(d)] [16].

Efficient OAM transfer from the driver to the plasma wake requires that the OAM difference $(\Delta \ell)$ between two photons separated by the plasma frequency $\omega_{p}$, given by $\Delta \ell=\ell\left(\omega+\omega_{p}\right)-\ell(\omega)=d \ell / d \omega \times \omega_{p}$, is an integer, which then corresponds to the $\mathrm{OAM}, \ell_{p} \in \mathbb{Z}$, acquired by the wakefield. This condition fixes the slope $\ell^{\prime}=$ $d \ell / d \omega$ of $\ell(\omega)$ to $\ell^{\prime}=\ell_{p} / \omega_{p}$. In the time domain, the physical meaning of this condition is that the temporal pitch $\tau_{h}$ of the LS intensity helix, given by $\tau_{h}=2 \pi\left|\ell^{\prime}\right|$ [16], needs to be an integer multiple of the plasma wave temporal period $\tau_{p}=2 \pi / \omega_{p}$, to ensure that the plasma wave excited by the final edge of the LS is in phase with the one previously excited by its starting edge.

Figure 2(a) shows the results of three-dimensional PIC simulations performed with OsIRIS $[19,20]$, illustrating a twisted plasma wave driven by a LS in these resonant conditions, with $\ell_{p}=1$. The simulation considers a preformed parabolic plasma channel to ensure stable propagation. The laser driver incorporates two different LG modes, with OAM levels differing by $\Delta \ell=1$ and frequencies (wave numbers) by $\Delta \omega=\omega_{p}\left(\Delta k=k_{p}\right)$, such that $\ell^{\prime}=1 / \omega_{p}$. The peak normalized vector potential of each mode is $a_{0}=0.75\left(a_{0}\right.$ relates to the peak laser
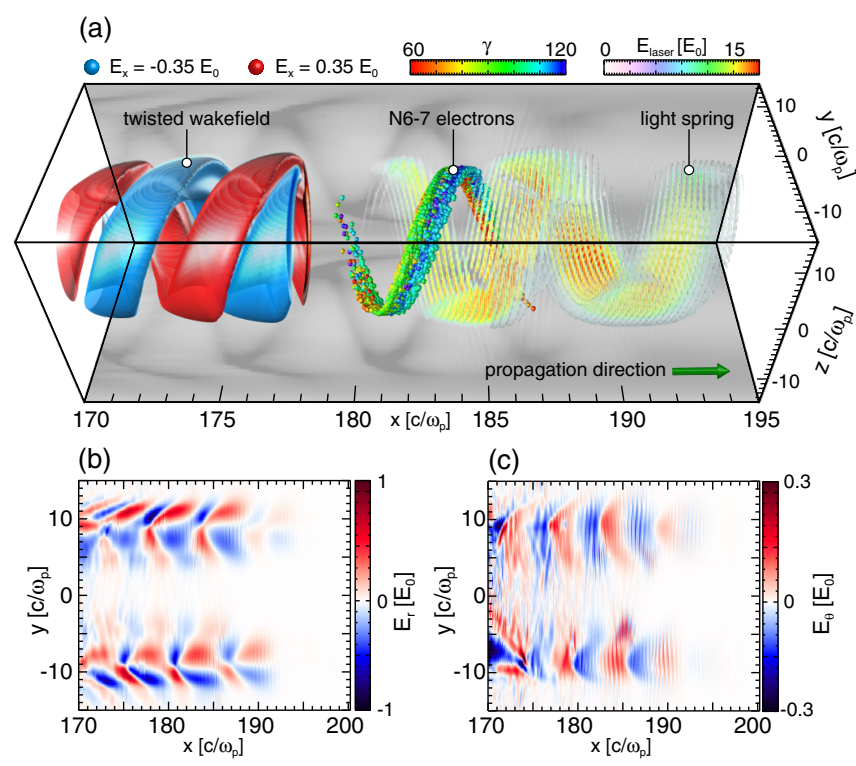

FIG. 2. Twisted plasma wakefields driven by a light spring moving in a preformed plasma doped with nitrogen. (a) (right) Rainbow colors display the electric field of the light spring. (left) Blue-red isosurfaces show the twisted longitudinal electric field structure of the wake excited by this LS in the underdense plasma. These surfaces are not displayed for $x \geq 178$ to avoid hiding the other plots. (middle) Spheres in rainbow colors correspond to ionization injected electrons from the inner (6th-7th) shells of nitrogen. (b)-(c) Slices of the corresponding radial and azimuthal electric fields in the plasma. Field values are normalized to the cold wave breaking limit $E_{0}=m_{e} c \omega_{p} / e$. intensity through $\left.a_{0}=8.6 \times 10^{-10} \lambda[\mu \mathrm{m}] I^{1 / 2}\left[\mathrm{~W} / \mathrm{cm}^{2}\right]\right)$. In addition a moving window with dimensions $36 \times 40 \times$ $40\left(c / \omega_{p}\right)^{3}$ and $2700 \times 200 \times 200$ cells was used. Each cell contains 10 particles (see Supplemental Material [21] for additional details). Because $\Delta \omega=\omega_{p} \quad\left(\Delta k=k_{p}\right)$ and $\Delta \ell=1$, this LS [green-yellow-red colors in Fig. 2(a)] is expected to excite a twisted wakefield with $\ell_{p}=1$. This agrees with the simulation, for instance, by considering the wake longitudinal electric field [blue and red surfaces in Fig. 2(a)]. More generally, simulations show that when $\ell^{\prime}$ is gradually increased, field structures with $\ell_{p}$ helical strands appear in the plasma when the resonance condition $\ell^{\prime}=$ $\ell_{p} / \omega_{p}$ is fulfilled.

The field structure of a twisted wakefield, similar to that shown in Fig. 2(a), can be captured analytically by a wake potential of the form $\phi=\phi_{0}(r) \cos \left(k_{p} \xi+\ell_{p} \theta\right)$, where $\phi_{0}(r)$ is the amplitude, $r$ is the distance to the axis, $\xi=$ $x-v_{\phi} t$ is the comoving frame variable with $v_{\phi} \simeq c$ being the wake phase velocity and $\theta$ the azimutal angle. The wake longitudinal electric field is then $E_{x}=-\partial \phi / \partial \xi=$ $k_{p} \phi_{0}(r) \sin \left(k_{p} \xi+\ell_{p} \theta\right)$. The OAM of the wake potential and electric fields thus coincide. Figure 2(a) and the analytical expression for $E_{x}$ also show that a closed path along the $\theta$ direction (for a fixed $\xi$ ) crosses positive and negative field regions. This contrasts with a non-OAM wakefield where positive and negative fields can only be accessed by following a path along $\xi$. This feature results from the modified topology of the plasma wave: while areas of constant field sign consist of a succession of totally disjoint "bubbles" in the wakefields created by standard Gaussian beams, here these areas form a set of intertwined helices, with a single continuous helix for each field value [Fig. 2(a)].

In contrast to planar wakefields, where the wave vector is constant and given by $k_{p} \mathbf{e}_{x}$, the twisted plasma wave vector is angle dependent, and given by $k_{p} \mathbf{e}_{x}+\ell_{p} / r \mathbf{e}_{\theta}\left(\mathbf{e}_{x}\right.$ and $\mathbf{e}_{\theta}$ are the unit vectors along $x$ and $\theta$, respectively). This allows exploring new acceleration regimes and generate new types of particle bunches. To investigate the new d.o.f. that now become available, we consider the Panofsky-Wenzel theorem [22], $\nabla_{\perp} E_{x}=\partial \mathbf{W}_{\perp} / \partial \xi$, with $\mathbf{W}_{\perp}=\mathbf{E}_{\perp}+c \mathbf{e}_{x} \times \mathbf{B}_{\perp}$, which relates the longitudinal and transverse wakefield components acting on a relativistic particle moving at $c$. Here, $\mathbf{E}_{\perp}$ and $\mathbf{B}_{\perp}$ are the transverse electric and magnetic wakefields, respectively. In addition to the radial focusing force, which appears since $E_{x}$ depends on $r$ [Fig. 2(b)], the Panofsky-Wenzel theorem predicts a new azimuthal field component [Fig. 2(c)], given by $(1 / r) \partial E_{x} / \partial \theta=\partial\left(E_{\theta}+B_{r}\right) / \partial \xi$, given by $E_{\theta}+B_{r}=\phi_{0}(r)\left(\ell_{p} / r\right) \sin \left(k_{p} \xi+\ell_{p} \theta\right)$. The new azimuthal wakefield component is a remarkable feature of twisted plasma waves, which strongly affects the dynamics of background plasma electrons and the dynamics of relativistic trapped particles.

The existence of a finite azimuthal wakefield, which is a result of the twisted wakefield topology, has far reaching 
consequences for the plasma dynamics and acceleration. Unlike planar wakefields, the longitudinal and azimuthal trajectories of the bunch particles are no longer independent. Their relation can be determined using Hamilton's equations. In an electrostatic wakefield, the canonical momentum along $x\left(\mathcal{P}_{x}\right)$ and $\theta\left(\mathcal{P}_{\theta}\right)$ correspond to the longitudinal (linear) momentum $\left(p_{x}\right)$ and angular momentum $\left(L_{x}\right)$, given by $\mathcal{P}_{x}=p_{x}=m_{e} c \gamma \beta_{x}$ and $\mathcal{P}_{\theta}=L_{x}=$ $r p_{\theta}=m_{e} c r \gamma \beta_{\theta}$, respectively. In these expressions, $\beta_{x}$ and $\beta_{\theta}$ are the longitudinal and azimuthal velocity components normalized to $c$. Hamilton's equations then read $d_{t} p_{x}=$ $-\partial_{x} \mathcal{H}$ and $d_{t} L_{x}=-\partial_{\theta} \mathcal{H}$, where $\mathcal{H}=m_{e} c^{2} \gamma+e \phi(r, \theta, \xi)$ is the Hamiltonian of a particle with charge $e$ and relativistic factor $\gamma$. Supplementing these equations with $d_{t} \mathcal{H}=\partial_{t} \mathcal{H}$ then leads to

$$
\frac{\Delta L_{x}}{\Delta p_{x}}=\frac{\ell_{p}}{k_{p}} .
$$

Equation (1) is a key result of the work. By establishing a proportionality relation between the angular and longitudinal momentum of the accelerated particles, Eq. (1) not only affects the dynamics of background plasma electrons, but has profound consequences on the dynamics of relativistic trapped particles, which are evident in the simulations. This proportionality relation is imposed by the wakefield topology. To clearly isolate the trapped particles, the simulation of Fig. 2 considers a small concentration of nitrogen, which allows for the occurrence of ionization injection from the inner 6th-7th atomic shells of the nitrogen. A helical particle bunch [rainbow colored spheres in Fig. 2(a)], with a single helical strand, then forms and accelerates to relativistic energies.

According to Eq. (1), for a given $\Delta p_{x}$, the angular momentum $\Delta L_{x}$ can only vary in jumps, multiples of $\ell_{p}$ at a fixed plasma density. Figure 3(a) shows the bunch particle distribution in the $p_{\theta}-p_{x}$ phase space, obtained from numerical simulation performed with different values of $\Delta \ell=\ell_{p}$. The slope of these particle distribution varies in discrete steps that indeed follow the theoretical prediction, given by $p_{\theta} / p_{x}=\ell_{p} /\left(k_{p} r\right)$. Here, $r \simeq r_{0}=w_{0} \sqrt{\left|\ell_{0}\right| / 2}$ is the radius where the LS intensity is maximum, with $\ell_{0}=$ $\langle\ell(\omega)\rangle$ the frequency-averaged value of $\ell$. The azimuthal particle motion then dominates the transverse particle dynamics because $p_{\theta} \gg p_{r}$. Twisted wakefields can also be exploited to generate beams characterized by comparable longitudinal and transverse momenta $\left(p_{x} \sim p_{\theta}\right)$ when $\ell_{p} \simeq r_{0} k_{p}$. Figure 3(a) (green region) illustrates this regime when $\ell_{p}=4$, for which $p_{\theta} \simeq p_{x} / 2$.

A remarkable consequence that follows from the quantization of the angular momentum expressed by Eq. (1) is that $\beta_{\theta}$ of trapped particles can only take a discrete set of values. The emergence of this unusual quantization rule can be directly linked to the twisted wakefield topology. In the limit where $\beta_{x}=v_{x} / c \simeq 1$ and the particle was initially at
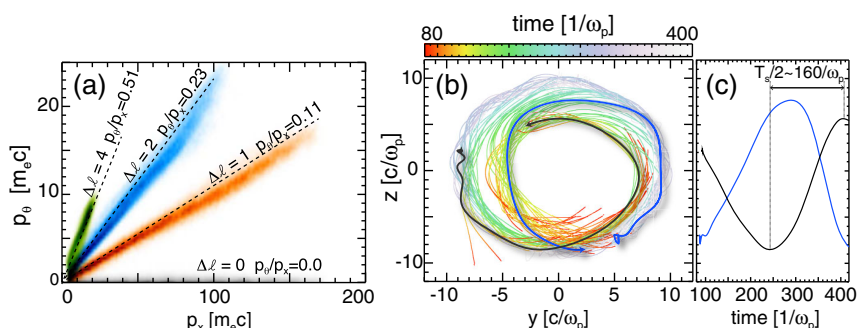

(d)
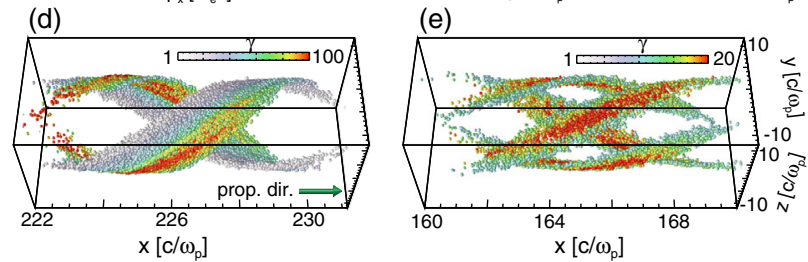

FIG. 3. Properties of relativistic vortex beams accelerated by twisted plasma waves. (a) $p_{\theta}-p_{x}$ phase space of vortex electron bunches in plasma waves with different OAM levels. The dashed lines show the prediction of Eq. (1) in each case. Note the negligible azimuthal momentum of trapped particles in the case where $\Delta \ell=0$ (gray distribution). (b) Helical trajectories of a random sample of ionization injected nitrogen electrons forming a vortex beam with $\ell_{p}=1$. The trajectories are colored according to the propagation time. (c) Trajectories of two electrons [shown in blue and red in (b)] along the propagation time. (d)-(e) Three-dimensional vortex particle bunches colored according to the energy.

rest, $\beta_{\theta} \simeq p_{\theta} / p_{x}=\ell_{p} /\left(k_{p} r_{0}\right)$. Thus, $\beta_{\theta}$ is quantized because it can only change by a multiple of $1 /\left(k_{p} r_{0}\right)$, as $\ell_{p}$ varies. The well-defined slopes of the phase-space regions in Fig. 3(a) already reflect this quantization rule. Figures 3(b) and 3(c) further confirm that trapped electrons perform helical trajectories with a period that agrees with theory. Figure 3(b) shows the trajectories of a random sample of trapped nitrogen electrons in the transverse $y-z$ plane for a twisted wakefield with $\ell_{p}=1$. Figure 3(c) shows that the spiralling period is $T_{s} \omega_{p} \simeq 320$, close to the theoretical estimate, given by $T_{s} \omega_{p}=2 \pi k_{p}^{2} r_{0}^{2} / \ell_{p}$, which yields $T_{s} \omega_{p} \simeq 353$.

Although purely classical, this quantization rule for $\beta_{\theta}$ is analogous to the OAM quantization of twisted rays of light as well as quantum vortex electron wave packets. To establish a parallel with twisted light, we consider the paraxial approximation, where $\mathbf{p}_{\perp} \ll p_{x}$, so that the energy $\left(E_{p}\right)$ of a relativistic particle is $E_{p} \simeq c p_{x} \simeq \gamma m_{e} c^{2}$. Hence, Eq. (1) leads to $L_{x} / E_{p} \simeq \ell_{p} / \omega_{p}$. The latter expression is analogous to the quantization of the ratio of angular momentum flux to energy flux for a LG beam [17], given by $M_{z}=\ell / \omega$. It also recovers the OAM quantization of vortex free electron quantum wave packet, given by $L_{x} / p_{x}=\ell / k_{x}$, where $k_{x}$ is the wave packet wave number [23]. These similarities suggest that trapped particles may be seen as a matter analogue of an OAM light beam and a classical matter analogue of a quantum electron wave packet. 
Figure 3(d), which illustrates the density distribution of the beam with $\ell_{p}=2$ [blue region in Fig. 3(a)] and Fig. 3(e) with $\ell_{p}=4$ [green region in Fig. 3(a)], shows the correspondence between the spatial and velocity distribution of the relativistic bunches: the number of helixes, given by $\ell_{p}$, is proportional to the angular momentum, given by the quantization rule $p_{\theta} / p_{x}=\ell_{p} /\left(k_{p} r\right)$, thus demonstrating that these beams constitute a new type of charged particle beams with a vortex spatial structure and with quantized levels of angular momentum.

A general feature of the acceleration in a twisted wake is that particles can also dephase along $\mathbf{e}_{\theta}$, thereby potentially lowering the dephasing length and energy gain in comparison to a planar wakefield. Figure 3(a) confirms this prediction, showing that the energy gain decreases for larger $\Delta \ell$. This effect can be mitigated due to a new and intrinsic property of twisted wakefields: as a LS propagates in the plasma, its intensity helix rotates azimuthally. This rotation enables us to adjust the wakefield phase velocity all optically. Remarkably, in some cases, the acceleration can even become stronger than in a planar wakefield.

While signatures for this rotation can be observed in a uniform plasma in the presence of self-guiding induced by the nonlinear plasma response, this effect becomes particularly clear, and its derivation simpler, in the presence of a preformed parabolic plasma channel, in the linear propagation regime. Figure 4(a) illustrates this azimuthal

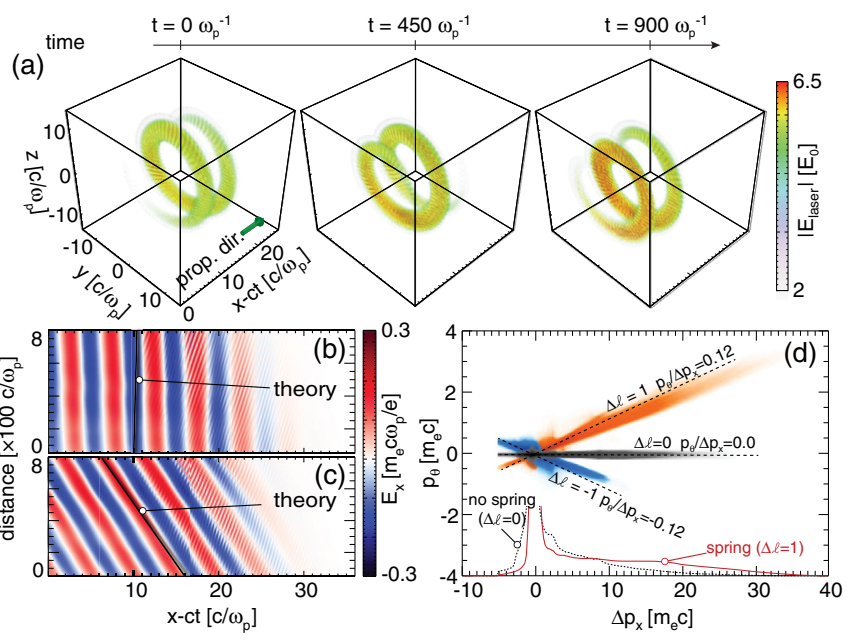

FIG. 4. Effects of the LS azimuthal rotation. (a) Spatiotemporal intensity profile of a LS propagating in a plasma channel, at different times in the propagation. (b)-(c) Evolution of the accelerating fields at $y \simeq 9 c / \omega_{p}$ and $z=0$, corresponding to the region around the radial maximum of the LS intensity, for a fixed $\ell_{0}>0$. The theoretical predictions of Eq. (2) are shown as black lines. In panel (b), the phase velocity of the wakefield is very close to $c>v_{g}$, due to the effect of the LS rotation. (d) $p_{\theta}-\Delta p_{x}$ phase space in twisted plasma waves with different OAM levels. The theoretical predictions of Eq. (1) are given by the black dashed lines. Solid red and point black curves, respectively, show the spectra of accelerated particles. rotation of the LS intensity helix, as it propagates in such a preformed plasma channel (see Supplemental Material [21] for the simulation details).

The channel counteracts diffraction by preventing the wave fronts to curve outwards. The angular momentum of a twisted light ray is preserved in the channel, causing the LS intensity profile to spin around its axis, in a direction determined by the helicity of the wave fronts, i.e., by the sign of $\ell_{0}$. Just as in a rotating screw, the laser pulse at a given radial position appears to move either backward or forward in the frame of the laser, depending whether this ray rotation is opposite to, or along the LS intensity helix, which direction is determined by the sign of $\ell^{\prime}=d \ell / d \omega$. Thus, when looking at a given longitudinal plane (along $x$ for fixed $y$ or $z$ ), the laser pulse envelope will appear to move either slower or faster than the linear group velocity in the plasma, depending on the relative sign of $\ell_{0}$ and $\ell^{\prime}$.

The resulting effective group velocity can be determined analytically (see Supplemental Material [21] for an explicit derivation),

$$
v_{g, \mathrm{LS}}=v_{g}+\frac{2 c^{2}}{k w_{0}^{2}} \ell^{\prime} \operatorname{sgn}\left(\ell_{0}\right)
$$

where $v_{g}$ is the usual group velocity in the plasma.

The plasma responds to this effective local group velocity of the LS. Hence, the correction term resulting from the rotation of the LS can be exploited to increase or decrease the wakefield phase velocity. Figures 4(b) and 4(c) show the evolution of the wakefields as a function of propagation distance when $\ell^{\prime}>0$ [Fig. 4(b)] and $\ell^{\prime}<0$ [Fig. 4(c)], for a fixed $\ell_{0}>0$, and confirm this prediction. This effect vanishes when $\ell^{\prime}=0$, and is thus an unique feature of twisted wakefields driven by the LS.

Since the energy gain of a group of externally injected particles depends on the wake phase velocity, this effect should modify the spectra of particles accelerated by twisted wakefields. This is illustrated on Fig. 4(d): the accelerated bunch reaches higher energies when $\ell^{\prime}$ and $\ell_{0}$ have the same sign (orange region), than when they are of opposite signs (blue region). For these parameters (i.e., keeping laser energy constant in all cases), Fig. 4(d) also demonstrates that the maximum energy and number of accelerated particles in the twisted wakefield (red solid line) exceeds those produced in a planar wakefield excited by a standard LG beam of same $\ell_{0}$ (black dashed line).

Most physical schemes studied so far to transfer angular momentum from lasers to plasmas have relied on circularly-polarized light, i.e., on the spin angular momentum of light [24-30], leading to strong magnetic fields, intense radiation bursts, and helical beam-plasma structures in dense plasmas exposed to superintense laser fields in the context of radiation pressure dominant acceleration [30]. In contrast, our work describes a new configuration where it is now the orbital angular momentum of a laser field that is 
transferred to an underdense plasma. This enables control of the wakefield topology, which can then be exploited to generate relativistic beams with unprecedented properties, beyond current possibilities, thereby significantly expanding the potential of plasma based acceleration. This configuration can have broad implications in different fields [14,31-38], from the generation of twisted $\mathrm{x}$-rays and intense magnetic field generation in plasmas $[39,40]$ to novel pathways to control laser-matter interactions, and perhaps manipulate the spin in compact plasma based spinpolarisers (e.g., due to the Sokolov-Ternov effect [41] or spin precession [42]). The required spatiotemporally shaped ultrashort laser beams can in principle already be obtained experimentally with simple helical mirrors such as those presented in Ref. [43]. Looking further ahead, advances in ultrafast optical metrology [44] and shaping [45] should soon provide access to advanced and programmable spatiotemporal control of ultrashort laser beams, and thus make it possible to tailor the topology of laser-plasma accelerators and hence access new intrinsic d.o.f. of the resulting high-energy particle beams.

We acknowledge the grant of computing time by the Leibnitz Research Center on SuperMUC. Work partially supported by the EU Accelerator Research and Innovation for European Science and Society (EU ARIES) Grant Agreement No. 730871 (H2020-INFRAIA-2016-1). J. V. acknowledges the support of FCT (Portugal) Grant No. SFRH/IF/01635/2015, and F. Q. the support from the European Research Council under the European Union's Horizon 2020 research and innovation programme (ERC Grant Agreement No. 694596).

[1] T. Tajima and J. M. Dawson, Phys. Rev. Lett. 43, 267 (1979).

[2] A. Modena, A. Dangor, Z. Najmudin, C. Clayton, K. Marsh, C. Joshi, V. Malka, C. Darrow, D. Neely, and F. Walsh, Nature (London) 377, 606 (1995).

[3] V. Malka, S. Fritzler, E. Lefebvre, M.-M. Aleonard, F. Burgy, J.-P. Chambaret, J.-F. Chemin, K. Krushelnick, G. Malka, S. P. D. Mangles, S. Najmudin, M. Pittman, J.-P. Rousseau, J.-N Scheurer, B. Walton, and A. E. Dangor, Science 298, 1596 (2002).

[4] A. Pukhov and J. Meyer-ter-Vehn, Appl. Phys. B 74, 355 (2002).

[5] W. Lu, C. Huang, M. Zhou, W. B. Mori, and T. Katsouleas, Phys. Rev. Lett. 96, 165002 (2006).

[6] W. Lu, M. Tzoufras, C. Joshi, F. S. Tsung, W. B. Mori, J. Vieira, R. A. Fonseca, and L. O. Silva, Phys. Rev. ST Accel. Beams 10, 061301 (2007).

[7] E. Esarey, C. B. Schroeder, and W. P. Leemans, Rev. Mod. Phys. 81, 1229 (2009).

[8] S. P. D. Mangles, C. D. Murphy, Z. Najmudin, A. G. R. Thomas, J. L. Collier, A. E. Dangor, E. J. Divall, P. S. Foster, J. G. Gallacher, C. J. Hooker, D. A. Jaroszynski， A. J. Langley, W. B. Mori, P. A. Norreys, F. S. Tsung, R. Viskup,
B. R. Walton, and K. Krushelnick, Nature (London) 431, 535 (2004).

[9] C. G. R. Geddes, Cs. Toth, J. van Tilborg, E. Esarey, C. B. Schroeder, D. Bruhwiler, C. Nieter, J. Cary, and W. P. Leemans, Nature (London) 431, 538 (2004).

[10] J. Faure, Y. Glinec, A. Pukhov, S. Kiselev, S. Gordienko, E. Lefebvre, J.-P. Rousseau, F. Burgy, and V. Malka, Nature (London) 431, 541 (2004).

[11] H. T. Kim, K. H. Pae, H. J. Cha, I J. Kim, T. J. Yu, J. H. Sung, S. K. Lee, T. M. Jeong, and J. Lee, Phys. Rev. Lett. 111, 165002 (2013).

[12] W. P. Leemans, A. J. Gonsalves, H.-S. Mao, K. Nakamura, C. Benedetti, C. B. Schroeder, Cs. Tóth, J. Daniels, D. E. Mittelberger, S. S. Bulanov, J.-L. Vay, C. G. R. Geddes, and E. Esarey, Phys. Rev. Lett. 113, 245002 (2014).

[13] S. G. Rykovanov, C. B. Schroeder, E. Esarey, C. G. R. Geddes, and W. P. Leemans, Phys. Rev. Lett. 114, 145003 (2015).

[14] J. Vieira and J. T. Mendonça, Phys. Rev. Lett. 112, 215001 (2014).

[15] J. T. Mendonça and J. Vieira, Phys Plasmas 21, 033107 (2014).

[16] G. Pariente and F. Quéré, Opt. Lett. 40, 2037 (2015).

[17] L. Allen, M. W. Beijersbergen, R. J. C. Spreeuw, and J. P. Woerdman, Phys. Rev. A 45, 8185 (1992).

[18] B. I. Cohen, A. N. Kaufman, and K. M. Watson, Phys. Rev. Lett. 29, 581 (1972).

[19] R. A. Fonseca et al., Lecture Notes in Computer Science (Springer, Berlin, Heidelberg, 2002), Vol. 2331.

[20] R. A. Fonseca, J. Vieira, F. Fiuza, A. Davidson, F. S. Tsung, W. B. Mori, and L. O. Silva, Plasma Phys. Controlled Fusion 55, 124011 (2013).

[21] See Supplemental Material at http://link.aps.org/ supplemental/10.1103/PhysRevLett.121.054801 for a detailed description of the PIC simulation parameters, and for an analytical derivation of the light spring dynamics in an external parabolic plasma channel.

[22] W. K. H. Panofsky and W. A. Wenzel, Rev. Sci. Instrum. 27, 967 (1956).

[23] K. Y. Bliokh, P. Schattschneider, J. Verbeeck, and F. Nori, Phys. Rev. X 2, 041011 (2012).

[24] I. Kostyukov, G. Shvets, and N. J. Fisch, Phys. Plasmas 9, 636 (2002).

[25] G. Shvets, N. J. Fisch, and J.-M. Rax, Phys. Rev. E 65, 046403 (2002).

[26] N. Naseri, V. Yu. Bychenkov, and W. Rozmus, Phys. Plasmas 17, 083109 (2010).

[27] T. V. Liseykina, S. V. Popruzhenko, and A. Macchi, New J. Phys. 18, 072001 (2016).

[28] Z. Lécz, A. Andreev, and A. Sery, Laser Part. Beams 34, 31 (2016).

[29] T.-P. Yu, A. Pukhov, Z.-M. Sheng, F. Liu, and G. Shvets, Phys. Rev. Lett. 110, 045001 (2013).

[30] M. Tamburini, T. V. Liseykina, F. Pegoraro, and A. Macchi, Phys. Rev. E 85, 016407 (2012).

[31] A. Denoeud, L. Chopineau, A. Leblanc, and F. Quéré, Phys. Rev. Lett. 118, 033902 (2017).

[32] A. Leblanc, A. Denoeud, L. Chopineau, G. Mennerat, Ph. Martin, and F. Quéré, Nat. Phys. 13, 440 (2017).

[33] J. Vieira, R. M. G. M. Trines, E. P. Alves, R. A. Fonseca, J. T. Mendonça, R. Bingham, P. Norreys, and L. O. Silva, Nat. Commun. 7, 10371 (2016). 
[34] J. Vieira, R. M. G. M. Trines, E. P. Alves, R. A. Fonseca, J. T. Mendonça, R. Bingham, P. Norreys, and L. O. Silva, Phys. Rev. Lett. 117, 265001 (2016).

[35] C. Brabetz, S. Busold, T. Cowan, O. Deppert, D. Jahn, O. Kester, M. Roth, D. Schumacher, and V. Bagnoud, Phys. Plasmas 22, 013105 (2015).

[36] X. Zhang, B. Shen, Y. Shi, X. Wang, L. Zhang, W. Wang, J. Xu, L. Yi, and Z. Xu, Phys. Rev. Lett. 114, 173901 (2015).

[37] C. Baumann and A. M. Pukhov, Quantum Electron. 47, 194 (2017).

[38] B. Izquierdo, R. J. Gray, M. King, R. J. Dance, R. Wilson, J. McCreadie, N. M. H. Butler, R. Capdessus, S. Hawkes, J. S. Green, M. Borghesi, D. Neely, and P. McKenna, Nat. Phys. 12, 505 (2016).
[39] Y. Shi, J. Vieira, R. Trines, R. Bingham, B. Shen, and R. Kingham, arXiv:1802.08582.

[40] R. Nuter, Ph. Korneev, I. Thiele, and V. Tikhonchuk, arXiv:1804.03574.

[41] A. A. Sokolov and I. M. Ternov, Sov. Phys. Dokl. 8, 1203 (1964).

[42] J. Vieira, C.-K. Huang, W. B. Mori, and L. O. Silva, Phys. Rev. ST Accel. Beams 14, 071303 (2011).

[43] D. P. Ghai, Appl. Opt. 50, 1374 (2011).

[44] G. Pariente, V. Gallet, A. Borot, O. Gobert, and F. Quéré, Nat. Photonics 10, 547 (2016).

[45] B. Sun, P. S. Salter, and M. J. Booth , Opt. Express 23, 19348 (2015). 